physician visits in baseline. TCZ users were less likely to be diabetic, use methotrexate in the baseline, and to be naïve to bDMARDS.

The crude incidence rate (IR) per 1000 patient-years for composite CVD among Medicare patients ranged from $13.3(95 \% \mathrm{Cl}: 11.1$ to 16.0$)$ for etanercept to 19.4 (95\% Cl: 16.3 to 20.9 ) for rituximab users. The crude incidence rate for pooled TNFi users was 16.4 (15.2-17.7). Compared to TCZ, the adjusted hazard ratios were $1.03(0.82-1.29)$ for abatacept, $1.25(0.96-1.61)$ for rituximab, $1.13(0.84-$ 1.52) for etanercept, $1.33(0.99-1.80)$ for adalimumab, and $1.57(1.21-2.05)$ for infliximab (figure 1). There were no significant differences in CVD risk between tocilizumab and any other biologic using MarketScan data. Results were robust in numerous subgroup analyses.

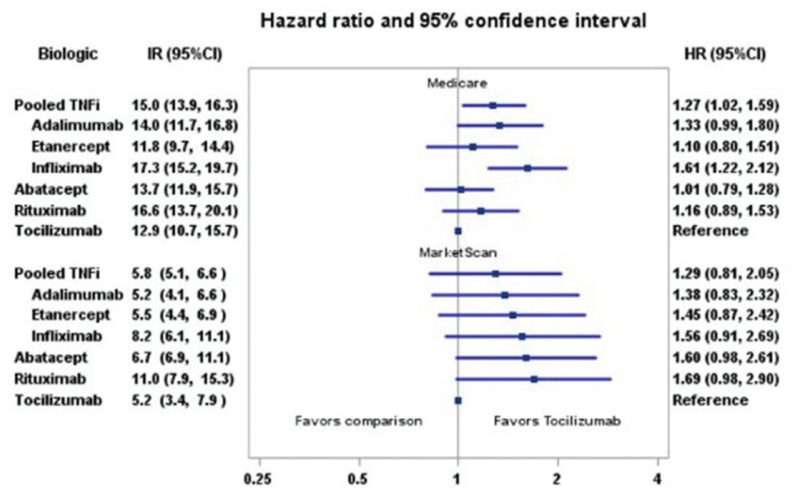

Abstract OP0193 - Figure 1. Incidence rates and adjusted hazard ratios of CVD events in RA patients

Conclusions: Consistent with findings of a recently completed safety trial in RA, tocilizumab was associated with a comparable CVD risk compared to etanercept, as well as a number of other RA biologics, in two large data sources.

Disclosure of Interest: F. Xie: None declared, H. Yun Grant/research support from: BMS, E. Levitan Grant/research support from: Amgen, Consultant for: Amgen, Novartis, P. Muntner: None declared, J. Curtis Grant/research support from: AbbVie, Amgen, BMS, Corrona, Janssen, Lilly, Myriad, Pfizer, Roche/Genentech, UCB, Consultant for: AbbVie, Amgen, BMS, Corrona, Janssen, Lilly, Myriad, Pfizer, Roche/Genentech, UCB

DOI: 10.1136/annrheumdis-2018-eular.5807

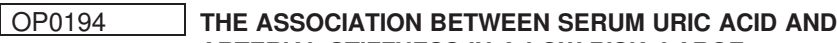 ARTERIAL STIFFNESS IN A LOW-RISK, LARGE POPULATION OF MIDDLE-AGED KOREAN}

J. Hwang ${ }^{1}$, J.H. Hwang ${ }^{2}$, Y. Eun ${ }^{3}$, H. Kim ${ }^{3}$, J. Lee ${ }^{3}$, J.K. Ahn ${ }^{4} .{ }^{1}$ Department of internal medicine, National Police Hospital; ${ }^{2}$ Center for health promotion, Samsung Medical Center, ${ }^{3}$ Department of medicine, Samsung Medical Center, Sungkyunkwan University School of Medicine; ${ }^{4}$ Department of internal medicine, Kangbuk Samsung Hospital, Sungkyunkwan University School of Medicine, Seoul, Korea, Republic Of

Background: Arterial stiffness occurs because of biologic ageing and arteriosclerosis, and is most commonly measured by pulse-wave velocity. Several studies have reported that high serum uric acid may contribute to the development of a number of metabolic and haemodynamic abnormalities, and multivariate analyses in epidemiologic studies have suggested that hyperuricemia is an independent risk factor for arterial stiffness in those with comorbidities such as diabetes, hypertension, and chronic kidney disease. However, there are few reports about the association between SUA and arterial stiffness in apparently healthy populations. Objectives: We aimed to investigate the association between serum uric acid (SUA) and arterial stiffness as evaluated by brachial ankle pulse wave velocity (baPWV) in a low-risk, large, middle-aged Korean population.

Methods: We conducted a cross-sectional study of 66,917 Koreans (38 170 men, 28747 women) who received yearly screening with available PWV and SUA results. None of the participants had coronary heart disease, diabetes, or hypertension. SUA was divided into quintiles for assessment of its association with baPWV by multiple linear regression analysis.

Results: The average SUA level was $5.23 \pm 1.4 \mathrm{mg} / \mathrm{dl}$, and SUA values were higher in men than in women $(6.1 \pm 1.2 \mathrm{mg} / \mathrm{dl}$ vs $4.1 \pm 0.8 \mathrm{mg} / \mathrm{dl})$. In multiple regression analysis, PWV was significantly higher in SUA quintiles 2-5 compared to the lowest group (reference) (coefficient=11.52, 18.19, 24.73, and $31.02 \mathrm{~cm} / \mathrm{s}$, respectively). In female subjects, the average difference $(\mathrm{cm} / \mathrm{s})$ of PWV between quintiles $2-5$ and quintile 1 of SUA was $13.1,22.9,34.6$, and 32.1, respectively.
Fully adjusted linear coefficient $\beta$ (S.E.) was $6.62(0.70)$ and $12.43(1.33)$ in all participants and female subjects, respectively $(p<0.001)$. In contrast, there was a Jshaped association between PWV and SUA quintile among males. When mod elled continuously, each $1 \mathrm{mg} / \mathrm{dl}$ higher SUA level was associated with 0.27 higher baPWV $(p<0.001)$ in the adjusted analysis.

Conclusions: These findings indicate that higher SUA levels could have an unfavourable impact on arterial stiffness as measured by baPWV in a low-risk large, middle-aged Korean population.

Disclosure of Interest: None declared

DOI: 10.1136/annrheumdis-2018-eular.1848

\section{OP0195 ROLE OF SEROPOSITIVITY ON MORTALITY IN RA AND THE IMPACT OF TREATMENT WITH DMARDS}

E. Alemao ${ }^{1}$, Z. Guo ${ }^{1}$, M.E. Weinblatt ${ }^{2}$, N.A. Shadick ${ }^{2} .{ }^{1}$ Bristol-Myers Squibb, Princeton; ${ }^{2}$ Brigham and Women's Hospital, Boston, USA

Background: Previous studies showed that RF positivity (+) in RA was associated with increased overall mortality, and that cause-specific mortality rates differed by autoantibodies. Anti-citrullinated protein antibodies (ACPAs) have been associated with cardiovascular death, and RF with death due to neoplasm and respiratory disease. ${ }^{1}$

Objectives: To evaluate the association of serostatus (in particular, antibody [Ab] titres) with mortality and its modification by DMARDs.

Methods: Administrative claims data from Optum Clinformatics Data Mart and Humana databases (2006-2016) were used. Inclusion criteria: 2 diagnosis codes for RA plus 1 DMARD prescription; age $>18$ years $(\mathrm{y}) ;>6$ months $(\mathrm{M})$ baseline (BL;+/-3 M from index date). Index date was the first test date for ACPA or RF (main analysis) or the DMARD prescription date (DMARD effect on mortality analysis). Patients (pts) with ankylosing spondylitis, Crohn's disease, lupus, psoriatic arthritis or ulcerative colitis at/before index date were excluded. Based on $B L A b$ test, pts were categorised into $\mathrm{Ab}$ status of $\mathrm{ACPA}+/-, \mathrm{RF}+/$-and double +/$A b+p t s$ were then categorised into 2 groups based on $A b$ titres. DMARD-exposed pts were categorised into biologic (b)DMARD (use of any bDMARD) and conventional (c)DMARD (use of a cDMARD but never a bDMARD) cohorts. Crude mortality rates per $1000 \mathrm{pt}-\mathrm{y}$, as well as adjusted analysis using traditional multivariate regression and disease risk score methods, were used. Covariates were age, sex, region, physician office visits in past $3 \mathrm{M}$, indicator variable for RA diagnosis before ACPA/RF testing, past hospitalisation, medication use (steroids, NSAIDs, salicylates), DMARD use and co-morbidities.

Results: A total of 53849 and 79926 pts with RA had evaluable ACPA and RF status, respectively. The average (SD) age was 61.4 (15.2) and 61.8 (15.6) y in the ACPA and RF cohorts, respectively. For both ACPA and RF, mortality rates were significantly higher in $\mathrm{Ab}+\mathrm{vs} \mathrm{Ab}-\mathrm{pts}$, and were highest in pts with the highest $\mathrm{Ab}$ titres (table 1). The hazard ratios (HRs) for mortality were highest in pts with double positivity (figure $1 A$ ). HRs were higher in $A b+v s A b-p t s$ exposed to cDMARDs. There was no difference in mortality between $A b+v s A b-$ pts in the bDMARD-exposed group (figure 1B).

Abstract OP0195 - Table 1 Crude mortality rates and HRs in patients categorised by serostatus

\begin{tabular}{|c|c|c|c|c|c|c|}
\hline & $\begin{array}{c}\text { Patients, } \\
\text { n }\end{array}$ & $\begin{array}{c}\text { Deaths, } \\
\mathbf{n}\end{array}$ & Pt-y & $\begin{array}{c}\text { Crude mortality, } \\
\text { incidence rate } / 1000 \\
\text { pt-y }(95 \% \mathrm{Cl})\end{array}$ & $\begin{array}{c}\text { Adjusted } \\
\text { HR } \\
(95 \% \mathrm{Cl})\end{array}$ & $\begin{array}{c}P \\
\text { value }\end{array}$ \\
\hline ACPA- & 36667 & 1798 & 126451 & $14.2(13.6-14.9)$ & 1.00 & \\
\hline ACPA+ & 17182 & 1276 & 57719 & $22.1(20.9-23.4)$ & $\begin{array}{c}1.48 \\
(1.37- \\
1.60)\end{array}$ & $<0.001$ \\
\hline $\begin{array}{l}\text { ACPA } \\
+ \text { +Group } \\
1^{\star}\end{array}$ & 8321 & 606 & 29518 & $20.5(18.9-22.2)$ & $\begin{array}{c}1.38 \\
(1.25- \\
1.52)\end{array}$ & $<0.001$ \\
\hline $\begin{array}{l}\text { ACPA } \\
+ \text { Group } \\
2^{*}\end{array}$ & 8861 & 670 & 28201 & $23.8(22.0-25.6)$ & $\begin{array}{c}1.60 \\
(1.45- \\
1.76)\end{array}$ & $<0.001$ \\
\hline RF- & 46376 & 2522 & 179247 & $14.1(13.5-14.6)$ & 1.00 & \\
\hline RF+ & 33550 & 2688 & 118583 & $22.7(21.8-23.5)$ & $\begin{array}{c}1.44 \\
(1.36- \\
1.53)\end{array}$ & $<0.001$ \\
\hline $\begin{array}{l}\text { RF } \\
+ \text { Group } \\
1^{*}\end{array}$ & 16758 & 1098 & 60393 & $18.2(17.1-19.3)$ & $\begin{array}{c}1.18 \\
(1.09- \\
1.27)\end{array}$ & $<0.001$ \\
\hline $\begin{array}{l}\text { RF } \\
+ \text { +Group } \\
2^{*}\end{array}$ & 16792 & 1590 & 58190 & $27.3(26.0-28.7)$ & $\begin{array}{c}1.79 \\
(1.51- \\
2.11)\end{array}$ & $<0.001$ \\
\hline
\end{tabular}

*Based on antibody titre: Group 1=lower titre; Group 2=higher titre 
A

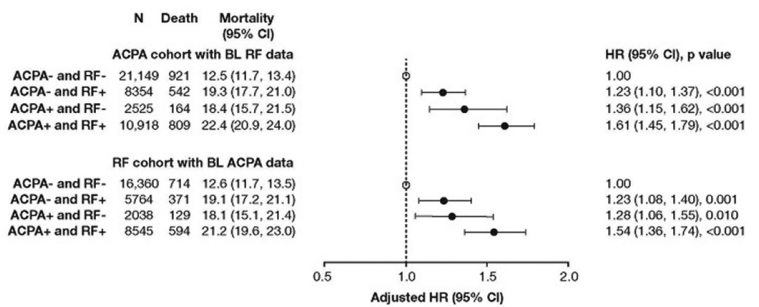

B

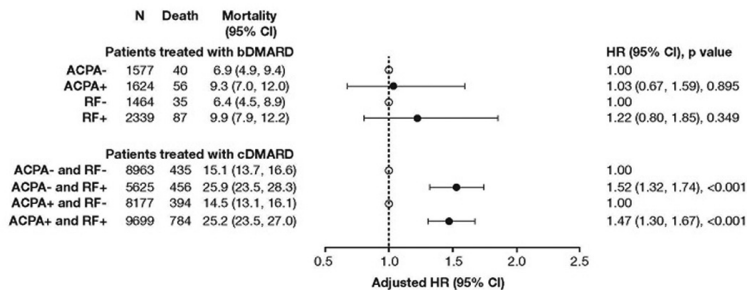

Abstract OP0195 - Figure 1 Mortality rates and HRs in patients categorised by (A) double ACPA and RF serostatus; (B) serostatus and DMARD exposure

Conclusions: Elevated ACPA and RF titres were independently associated with increased mortality among pts with RA. The associations between ACPA/RF and mortality persisted in those treated with CDMARDs but not with bDMARDs. Further studies are warranted to evaluate the effect of bDMARDs on mortality in seropositive pts.

\section{REFERENCE:}

[1] Ajeganova S, et al. Ann Rheum Dis 2016;75:1924-32.

Disclosure of Interest: E. Alemao Shareholder of: Bristol-Myers Squibb, Employee of: Bristol-Myers Squibb, Z. Guo Employee of: Bristol-Myers Squibb, M. Weinblatt Grant/research support from: Amgen, Bristol-Myers Squibb, Crescendo Bioscience, Sanofi, Consultant for: Amgen, Bristol-Myers Squibb, Crescendo Bioscience, AbbVie, Lilly, Pfizer, Roche, Merck, Samsung Novartis, N. Shadick Grant/research support from: BRASS registry, Amgen, Bristol-Myers Squibb, and Mallinckrodt, Consultant for: Bristol-Myers Squibb DOI: 10.1136/annrheumdis-2018-eular.1655

\section{OP0196 SAFETY AND EFFICACY OF IMMUNE CHECKPOINT INHIBITORS IN PATIENTS WITH CANCER AND PREEXISTING AUTOIMMUNE DISEASES: A NATIONWIDE MULTICENTER RETROSPECTIVE STUDY}

A. Tison ${ }^{1}$, G. Quere ${ }^{1}$, L. Misery ${ }^{1}$, E. Funck-Brentano ${ }^{2}$, F.-X. Danlos ${ }^{2}$, B. Bonniaud ${ }^{3}$,

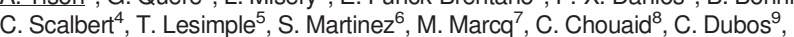
F. Brunet-Possenti ${ }^{2}$, C. Stavris ${ }^{10}$, L.Y. Chiche ${ }^{10}$, D. Cornec ${ }^{1}$, M. Kostine ${ }^{11}$, on behalf of Groupe de Cancérologie Cutanée (GCC), Groupe Français de PneumoCancérologie (GFPC), Club Rhumatisme et Inflammation (CRI). ${ }^{1} \mathrm{CHU}, \mathrm{Brest}{ }^{2} \mathrm{AP}$ HP, Paris; ${ }^{3} \mathrm{CHU}$, Dijon; ${ }^{4} \mathrm{CHU}$, Lille; ${ }^{5} \mathrm{Centre}$ anti-cancer, Rennes; ${ }^{6} \mathrm{CH}$, Aix $;{ }^{7} \mathrm{CH}$ Vendée, La Roche sur Yon; ${ }^{8} \mathrm{CHU}$, Créteil; ${ }^{9}$ Centre Baclesse, Caen; ${ }^{10}$ Européen, Marseille; ${ }^{11} \mathrm{CHU}$, Bordeaux, France

Background: Immune Checkpoint Inhibitors (ICI) have revolutionised the management of several cancers, enhancing the anti-tumoral immune response. However they are responsible for many Immune Related Adverse Effects (IRAE), and therefore most patients with Preexisting Autoimmune Diseases (PAD) have been excluded from clinical trials.

Objectives: The aim of this study was to evaluate the safety and efficacy of $\mathrm{ICI}$ in patients with PAD.

Methods: Three national expert networks, focusing respectively on skin cancers, thoracic cancers and inflammatory diseases participated in this study. All patients who received an ICI despite a PAD in clinical practice were included in this nationwide retrospective study.

Results: 112 patients were included: 64 men (57.1\%), median age 66.5. Most patients received an anti-PD1 or anti-PD-L1 drug (84.8\%). Main cancer types were melanoma ( $n=66,58.9 \%)$ and Non-Small Cell Lung Carcinoma (NSCLC) $(n=40 ; 35.7 \%)$. Median follow-up was 8 months [0-52]

Most frequent PAD were psoriasis and psoriatic arthritis $(27.6 \%)$, rheumatoid arthritis $(17.8 \%)$, inflammatory bowel disease $(12.5 \%)$, spondyloarthritis $(4.5 \%)$, lupus $(6.3 \%)$, polymyalgia rheumatica and/or giant-cell arteritis (6.3\%). 24 patients $(21.6 \%)$ were receiving an immunosuppressive therapy (IS) at $\mathrm{ICI}$ initiation (including steroids in 15, sDMARD in 10 and rituximab in 1). 37 patients (33\%) had an active disease.

PAD flares were frequent $(n=47 ; 42 \%)$ and $30.4 \%$ of them were severe (grade CTCAE 3-4). 26 patients (56.5\%) received an IS treatment for a flare (22 received steroids and 7 a DMARD). Other IRAEs not related to the PAD occurred in 43 patients $(38.4 \%), 41.5 \%$ were severe. 23 patients $(56.1 \%)$ required an IS (including a DMARD in 4). 36 patients (32.1\%) discontinued ICI temporarily or definitively because of a flare or an IRAE. One patient died due to an IRAE.

Concerning the anti-tumoral response, the Overall Response Rate (ORR) was $48.3 \%$ for melanoma and $53.8 \%$ for NSCLC. The median Progression Free Survival (PFS) was 12.4 months for melanoma and 9.7 for NSCLC. Median overall survival (OS) was not reached in any group. PFS and OS were shorter in patients receiving an $I S$ treatment at $I C l$ initiation $(p=0.007$, figure $1 A$, and $p=0.003$, respectively). PFS and OS were longer in patients who experienced a PAD flare or other IRAE, but this gain was lost when an IS was used to treat the flare/IRAE $(p=0.008$, figure $1 B$, and $p=0.01$, respectively). Conversely, this gain was not impacted with $\mathrm{ICI}$ discontinuation.
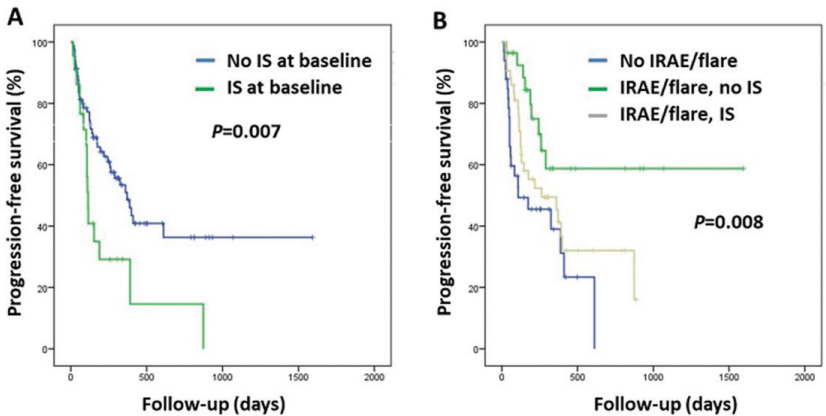

Conclusions: PAD flares and other IRAEs are frequent during $\mathrm{ICI}$ therapy and may be severe. The OS, ORR and PFS seem high in patients with PAD. The occurrence of a flare/IRAE is associated to a better outcome, gain lost when IS are used, while $\mathrm{ICI}$ discontinuation has no impact on PFS. Further prospective studies are needed to confirm our findings.

Disclosure of Interest: None declared

DOI: 10.1136/annrheumdis-2018-eular.5840

\section{OP0197 RHEUMATIC AND MUSCULOSKELETAL ADVERSE EVENTS ASSOCIATED WITH IMMUNE CHECKPOINT INHIBITORS: DATA MINING OF THE US FOOD AND DRUG ADMINISTRATION ADVERSE EVENT REPORTING SYSTEM}

X. Pundole ${ }^{1}$, M. Sarangdhar ${ }^{2}$, M.E. Suarez-Almazor ${ }^{3} .{ }^{1}$ The University of Texas MD Anderson Cancer Center, Houston; ${ }^{2}$ Division of Biomedical Informatics, Cincinnati Children's Hospital Medical Center, Cincinnati; ${ }^{3}$ Department of General Internal Medicine, Section of Rheumatology and Clinical Immunology, The University of Texas MD Anderson Cancer Center, Houston, USA

Background: Immune-modulating monoclonal antibodies directed against immune checkpoints ((cytotoxic T lymphocyte-associated antigen 4 (CTLA-4), programmed cell death-1 receptor (PD-1) and its ligand PD-L1)), have demonstrated tremendous promise in the treatment of diverse solid tumour types, including melanoma, non-small cell lung cancer, among others and have improved survival rates of these cancer patients. However, these advances have created a new set of challenges in identifying and managing toxicities.

Objectives: To identify emerging trends of rheumatic and musculoskeletal adverse events by immune checkpoint inhibitor $(\mathrm{ICl})$ treatment in the US Food and Drug Administration (FDA) Adverse Events Reporting System (FAERS).

Methods: We used AERSMine, an open-access web based application to mine the FAERS database from the first quarter (Q1) of 2011 to the third quarter (Q3) of 2017 , approximately 7.1 million patients. Measures of disproportionality were calculated using well-established pharmacovigilance metrics, Relative Risks (RR) and safety signals (information component, (IC)), in a subset of patients with a cancer diagnosis. Terminology used for categorization of adverse events was as included in the FAERS. Fisher's exact test was used to determine significant adverse event differences by $\mathrm{ICl}$ treatment and age.

Results: We identified 30939 unique patients with cancer and reports of immune checkpoint inhibitor associated toxicities. More than half of these reports were in relation with anti PD-1 inhibitors. Statistically significant adverse events associated with $\mathrm{ICl}$ therapy identified as toxicity signals with different agents included: NIVOLUMAB: myositis $(n=102 ; R R, 1.35 ; p<0.01 ; I C, 0.43)$, rheumatoid arthritis $(n=67 ; R R, 1.52 ; I C, 0.61)$, psoriatic arthropathy $(n=20 ; R R, 1.93, I C, 0.95)$, 